\title{
Qualité de l'évaluation en multimédia : vers un espace de classification des procédés d'interactivité
}

Jean-Marc Laubin, Alain Durand et Sylvie Leleu-Merviel

\section{(2) OpenEdition}

Journals

Édition électronique

URL : http://journals.openedition.org/communicationorganisation/2194

DOI : 10.4000/communicationorganisation.2194

ISSN : 1775-3546

Éditeur

Presses universitaires de Bordeaux

Édition imprimée

Date de publication : 1 mai 1999

ISSN : 1168-5549

Référence électronique

Jean-Marc Laubin, Alain Durand et Sylvie Leleu-Merviel, "Qualité de l'évaluation en multimédia : vers

un espace de classification des procédés d'interactivité », Communication et organisation [En ligne],

15 | 1999, mis en ligne le 19 décembre 2012, consulté le 19 avril 2019. URL : http://

journals.openedition.org/communicationorganisation/2194; DOI : 10.4000/

communicationorganisation.2194

Ce document a été généré automatiquement le 19 avril 2019

(c) Presses universitaires de Bordeaux 


\title{
Qualité de l'évaluation en multimédia : vers un espace de classification des procédés d'interactivité
}

\author{
Jean-Marc Laubin, Alain Durand et Sylvie Leleu-Merviel
}

\section{Introduction}

1 Les évolutions sociales, culturelles, techniques, comportementales, etc. ont généralisé le principe de l'évaluation des activités humaines. Cette évaluation renvoie implicitement au concept vague de "qualité » du résultat et/ou de la production correspondante. L'industrialisation de la majeure partie des dispositifs de production a renforcé ce souci, jusqu'à en faire un critère clef de mesure des performances. La rationalisation parallèle de l'ensemble du processus industriel a imposé une formalisation rigoureuse du concept qualité, permettant une quantification, c'est-à-dire une mesure objective, des performances. Ainsi, la qualité désigne l'ensemble des propriétés et caractéristiques d'un produit ou service qui lui confèrent l'aptitude à satisfaire des besoins exprimés ou implicites. Il est à noter qu'un produit est un résultat, concret ou abstrait, d'une production humaine liée à une activité quelconque. Par conséquent, un service est un produit.

2 Dans ce contexte, la médiocrité reconnue d'une grande partie des produits multimédia actuels constitue un frein majeur au développement du marché. En effet, le client potentiel, échaudé par une première acquisition décevante - quoiqu'assez onéreuse décide souvent de ne pas réitérer l'expérience. Il s'agit typiquement ici de produits ne répondant pas aux besoins des utilisateurs potentiels. Ainsi l'économie de ce secteur reste-t-elle très embryonnaire, en dépit de toutes les prévisions optimistes renouvelées chaque année. 
3 De plus, au moment de l'acte d'achat, très peu d'informations fiables sont fournies au client quant aux caractéristiques de l'objet qu'il envisage d'acquérir. Comme le remarquent Claire Schéol et Patrick Pognant, « on a l'impression - surtout lorsque l'on connait la réalité des contenus - que les descriptifs hyperboliques et emphatiques représentent une projection idéale de ce que les éditeurs rêvent que leurs titres soient. Les seules données objectives concernent l'indication des volumes. Mais là encore, la formulation superlative resurgit : il ne s'agit jamais de cinq cents écrans mais toujours de plus de cinq cents écrans ». D'où la conclusion des auteurs: "il serait temps d'offrir au chaland des indications débarrassées de leur prisme fantasmatique au profit d'une réelle valeur informative ».

Les professionnels de la production et/ou de l'édition ne sont malheureusement pas plus fournis en outils d'évaluation d'un projet. Les décisions se prennent par conséquent « en aveugle ", sans disposer de beaucoup d'éléments concrets de description concernant le produit à naître. De ce fait, on constate d'ores et déjà une sorte de standardisation de la production vers des schémas "sans risques ", parce que bien identifiés. Ainsi le recours à la structure arborescente est-il quasiment généralisé, alors qu'elle est très loin de constituer la seule forme d'écriture possible.

De l'ensemble de ces observations, il résulte que toute tentative de clarification et/ou de classification s'avère favorable à l'organisation d'un secteur qui pèche par son désordre. L'un des éléments prépondérants du multimédia, qui le distingue des autres formes d'édition de contenus (livres, supports audiovisuels, etc.) est l'interactivité. Dans cet article, ce paramètre clef est adopté comme facteur discriminant de classification des applications.

\section{Caractérisation préalable des documents interactifs}

\section{Définition de la notion de document}

6 La préparation d'un contenu informationnel, dans le but de le mettre à disposition par consultation interactive, consiste à sélectionner les données retenues, et à les organiser, c'est-à-dire à définir une structure élaborée comportant un ensemble de sous-parties, elles-mêmes décomposables jusqu'au niveau de la donnée élémentaire. L'appellation « document » désigne précisément cette structure organisationnelle. Ainsi, un document est une structure organisée de parties informationnelles de niveau moindre.

7 Il est utile d'ajouter à cette définition la précision suivante : un document est un contenu informationnel pouvant être supporté par un médium. En ce sens, une cassette vidéo, un livre, une toile... ne sont pas des documents mais des supports. En revanche, un film, un texte, une peinture... sont des documents. Ils sont indépendants du support de consultation et/ou de diffusion. Ils peuvent être consultables, en tout ou partie, sur un livre, sur une toile, sur un ordinateur, sur un réseau, etc. Le terme de document désigne ainsi la structure qui régit l'organisation de sous-parties constituantes, et ceci en totale indépendance vis-à-vis de l'objet physique réalisé en final. 


\section{Finalité des documents}

8 Au-delà de leur définition et de leur description fonctionnelle, la caractéristique majeure des documents concerne les objectifs que remplissent ces objets, leurs spécificités, c'est-àdire en définitive leur finalité.

9 La finalité du processus - comme dans tout problème de communication - consiste à exposer une cible à un message (i.e. un ensemble organisé de données sensorielles), dans un objectif donné, c'est-à-dire dans le but de produire un effet déterminé sur le récepteur. Toutefois, ce problème classique des sciences de l'information est ici compliqué par l'intégration des modalités propres à l'établissement d'échanges interactifs.

\section{Identification des tâches contribuant à la conception de documents}

\section{Approche scénistique}

10 La scénistique a été introduite dans de précédents travaux. Le lecteur pourra se reporter à la thèse d'habilitation à diriger des recherches de Sylvie Leleu-Merviel, la thèse d'Alain Durand ou l'ouvrage de Sylvie Leleu-Merviel à paraittre aux éditions Hermes en 1999 (cf. bibliographie), pour en prendre connaissance dans le détail. La scénistique est une méthodologie d'aide à la conception de document. Sa mise en œuvre s'appuie successivement sur la diégèse, le scénario, la scenario, la scénique et la mise en situation.

11 Définition : la diégèse est tout ce qui appartient, dans l'intelligibilité du document, au monde supposé ou proposé par le récit. C'est un monde virtuel, peuplé d'entités (lieux, personnages, objets, etc.), et régi par des lois internes. (La diégèse a déjà été définie par Étienne Souriau dans les années cinquante, les entités sont des objets, choses ou personnages qui participent à l'évolution ou à la description de l'environnement.)

La diégèse est l'univers, le monde virtuel dans lequel se déroule l'histoire : elle comprend les lieux, les personnages; elle contient le potentiel scénaristique (elle sert d'assise à l'élaboration du scénario). Elle définit chaque élément pertinent du monde supposé par le document.

Le scénario ne représente pas la structure proposée à la perception de l'utilisateur, mais modélise la structure événementielle profonde. Il a pour rôle de construire la narration : le scénario représente en ce sens «l'histoire » que l'on rencontre. Mais il y a une grande différence entre construire la logique des péripéties et organiser les constituants du récit lui-même.

Définition : la scénation désigne la structure organisée d'événements et/ou d'états avec lesquels le lecteur est effectivement mis en interaction. Elle est constituée d'un ensemble de fragments extraits du scénario. Lorsque la conception du scénario et celle du modèle scénationnel sont achevées on dispose du produit virtuel dans son intégralité.

Définition : la scénique désigne le processus permettant de transposer le texte en une réalité concrète: elle résulte de choix esthétiques, de contraintes pratiques ou financières, etc. 

télévision, cognition, page 132, où l'on comprend au fil du texte un sens proche de celui proposé ici, sans que Michel Colin n'en ait donné une définition précise. Par ailleurs, la notion de fragment rapproche cette définition de la pensée d'Eisenstein, lorsqu'il parle de prélèvement sur un réel agencé visant un montage productif. Dans le premier cas, l'utilisateur ne dispose d'aucune possibilité d'intervention sur l'enchaînement structurel des séquences qui lui sont proposées. La scénation résulte donc d'un choix délibéré de l'auteur, qui détermine une structure figée de récit. En revanche, dans le second cas, ce sont les actions de l'utilisateur, ses choix successifs et son comportement qui élaborent la scénation en temps réel, au fur et à mesure de l'interaction. Par conséquent :

23 - l'auteur n'a pas de contrôle direct sur la scénation,

24 - chaque session d'utilisation donne lieu à une scénation différente pour un même utilisateur,

Ainsi, en l'absence de fonctionnalités interactives, la scénation est déterministe et résulte de la conception de l'auteur (éventuellement du montage qui achève la réalisation). Dans le cas d'un produit interactif, la scénation n'est pas déterminée à l'avance et s'élabore au cours de la session interactive. Elle peut être confondue avec le "parcours exploratoire " de l'utilisateur au sein de la structure définie par le scénario. L'influence des modalités d'interactivité se traduit par des processus opposés de construction de la scénation d'un récit. 


\section{Identification de différents schémas de scénation}

\section{Niveau zéro : audio-vision}

En s'appuyant sur le concept de scénation proposé ci-dessus, on peut tenter une identification des différents modes scénationnels, répertoriés à partir d'une analyse des documents interactifs existants.

Le niveau zéro d'interactivité, celui pour lequel il n'y a pas d'interaction scénationnelle, correspond à un mode de diffusion linéaire continue. L'usager ne peut que suivre le discours qui se déroule dans le temps, sans pouvoir intervenir autrement qu'en abandonnant (comme on quitte une salle de cinéma ou comme on éteint la télévision). Les fragments de données sont ici enchaînés en séquence. On qualifie ce niveau zéro de procédé d'interactivité du type "audio-vision» (c'est-à-dire audition et visionnage simultanés), par analogie avec le cinéma ou la télévision. Notons que, malgré l'absence d'interactivité matérielle et conversationnelle, il s'établit bien une interactivité communicationnelle cognitive et/ou émotionnelle.

\section{Niveau un : lecture}

Le niveau un d'interaction scénationnelle satisfait à un mode de diffusion linéaire interruptible. Comme avec un magnétoscope, on peut revenir en arrière et rediffuser un passage.

Comme avec un livre, on peut sauter les premiers fragments et commencer par le milieu. Mais la structure globale reste fondamentalement séquentielle. Par analogie avec les autres media cités, ce niveau un de procédé d'interactivité est qualifié de type «lecture ». Il permet à l'utilisateur d'adopter son propre rythme et de s'affranchir d'une inscription déterminée dans le temps. Toutefois, le contrôle de la diffusion des contenus reste très faible.

\section{Niveau deux : navigation}

Le niveau deux permet d'enchaîner les unités d'informations (figées et insécables) suivant des parcours nombreux et divers parmi lesquels l'utilisateur est amené à choisir, mais dont les occurrences ont toutes été programmées par le scénariste. L'usager se promène dans les contenus, il se déplace, il voyage : c'est l'interaction de type «navigation». Mais tous les trajets possibles ont été prédéterminés. La forme de structure généralement adoptée pour ce genre d'application est l'arbre, ou arborescence.

\section{Niveau trois : consultation}

Pour le niveau trois, les unités d'informations insécables adoptent une structure indexée de type matricielle, dont chaque composante correspond à un item ou un identificateur précis. La lecture est alors séquentielle par cellule, chaque cellule étant repérée par un ou plusieurs index. L'utilisateur est adressé à la cellule de son choix par un processus de requête, souvent extrêmement formalisé. Ce niveau correspond à un procédé 
d'interactivité de type "consultation", ainsi que l'on consulte un horaire de chemin de fer ou un annuaire téléphonique (on ne les lit heureusement pas!).

\section{Niveau quatre : exploration}

À ce niveau, l'utilisateur ne se laisse plus porter par des parcours prédéterminés, mais des outils de structuration très puissants, tels que les réseaux de liens, permettent de générer des parcours totalement individualisés et pas forcément tous prévus par le concepteur. Ce type d'interaction scénationnelle, qualifié «d'exploration", convient tout particulièrement aux documents de nature encyclopédique.

\section{Niveau cinq : visite virtuelle}

Enfin, au niveau cinq n'intervient plus aucune diffusion séquentielle par morceaux. Il n'y a aucune modification de la diffusion qui ne soit liée à une action de l'utilisateur. En l'absence d'action, le programme reste délibérément figé. L'usager se trouve confronté à un espace continu d'interaction. Ce genre d'application, où les données obéissent à un modèle objet, correspond entre autres aux "visites guidées virtuelles", où les déplacements de l'utilisateur conditionnent l'affichage à chaque instant. C'est le type d'interaction que l'on trouve par exemple dans la partie jeu de « Versailles : complot à la cour du Roi Soleil ». Notons toutefois que, dans ce produit, la scénation est en visite virtuelle quant à l'image, mais en simple audition (cas particulier strictement sonore de l'audio-vision) pour le son musical, en navigation arborescente pour les échanges dialogues et en lecture pour les textes. Cet exemple montre par conséquent que le type de scénation peut être différent suivant le medium envisagé au sein d'une même application.

\section{Générativité des données}

\section{Définition des données}

La préparation d'un document consiste à sélectionner les données retenues et à les organiser en une structure déterminée. Par ailleurs, la scénation définit la manière dont l'utilisateur peut naviguer au travers des fragments de document qu'il consulte. On voit que la nature des données et la forme qui les structure conditionnent étroitement les modes scénationnels autorisés par le programme.

Mais pour pouvoir avancer davantage, il convient d'examiner plus précisément les différents types de données que peut comporter un document.

Une donnée est un enregistrement numérique de certains attributs d'un objet ou d'un événement. Ainsi, la donnée est ce qui est mémorisé par le programme.

\section{Classe de données fixes : niveau zéro}

À partir de l'analyse effectuée précédemment, on peut définir une première classe de données qualifiées de fixes.

Il s'agit des données, codées dans le programme, qui donnent elles-mêmes lieu à des opérations permettant leur manifestation, c'est-à-dire leur «affichage » sous une forme 
directement perceptible sensoriellement par l'utilisateur de l'hyperdocument. Ainsi, le tenseur de bits codant une image plane est directement converti en image perceptible visuellement, dans l'exemple cité précédemment. De plus, le regroupement des données en unités d'informations discrètes est invariable, figé pour toute utilisation ultérieure du document.

\section{Classes de données génératives}

$\mathrm{Au}$ contraire, les systèmes les plus sophistiqués techniquement peuvent faire en sorte que les éléments qui se manifestent médiatiquement par un affichage perceptible sensoriellement ne soient pas les données codées en mémoire. Ainsi que le souligne JeanPierre Balpe [BAL 90]: «Le texte que le lecteur parcourt sur un écran de lecture, le dessin qu'il regarde peuvent fort bien n'exister nulle part dans l'espace de mémorisation de l'ordinateur. Des programmes spécialisés peuvent, en fonction des besoins spécifiques ou à des fins particulières, créer en temps réel le texte qu'il est en train de lire, produire le dessin qu'il est en train de regarder."

On entre ainsi dans la classe de données dites génératives, c'est-à-dire disposant de fonctions permettant de générer les éléments proposés à la scénation. Alors que, pour la classe précédente, les données restaient strictement déclaratives, elles deviennent ici procédurales.

Cependant on distingue plusieurs niveaux de données génératives.

\section{Variables d'état définies initialement par l'application : Niveau un}

Lorsque des données sont générées par l'application, le premier degré de complexité consiste à définir le mode de génération, les paramètres qui feront évoluer les données (paramètres qui sont des variables d'état), avant que ne débute l'usage réel du document. Lorsque ces paramètres d'état sont déterminés par l'application elle-même, cette dernière a une générativité de niveau un. La détermination des variables d'état peut résulter par exemple d'un tirage pseudo-aléatoire. Ce niveau de générativité est la " générativité initiale simple ».

\section{Variables d'état définies initialement par l'utilisateur : Niveau deux}

Lorsque ce sont des actions de l'utilisateur qui permettent de définir les conditions initiales de la génération des données, le degré de complexité augmente. C'est pourquoi, ce niveau, qualifié de "générativité initiale utilisateur ", se trouve être le deuxième degré de complexité de classement des applications à données génératives. Il concerne encore les documents dont génération et paramètres d'évolution des données sont déterminés avant l'usage réel du document par l'utilisateur. Pour un document pédagogique, les réponses fournies à un questionnaire à choix multiple préalable, peuvent déterminer une génération de leçons, ou d'exercices. Ce document sera alors d'une générativité de niveau deux.

Ces deux niveaux ne s'appliquent pas aux documents dont les variables d'état peuvent être modifiées durant leur utilisation. 


\section{Variables d'état modifiées par le programme pendant l'usage de l'application : Niveau trois}

Ainsi, le troisième degré de complexité définit le niveau de "générativité dynamique simple ». Il concerne les documents à données génératives dont les variables d'états peuvent être modifiées au cours de leur utilisation, mais ces modifications sont engendrées uniquement par l'application elle-même. L'application dispose alors d'un modèle d'évolution dynamique des variables d'état, implémenté dans le programme. Le modèle exploite des données qui sont toutes indépendantes des actions de l'utilisateur.

\section{Variables d'état modifiées par l'utilisateur pendant l'usage de l'application : Niveau quatre}

Par extension, la "générativité dynamique utilisateur» concerne les documents à données génératives où l'utilisateur peut modifier les variables d'état d'évolution pendant la lecture du document. Comme dans le cas précédent, l'application dispose d'un modèle d'évolution dynamique des variables d'état. Leur modification en cours de session interactive s'effectue suivant des critères parmi lesquels figure, cette fois, les interactions avec l'utilisateur. On est passé d'un modèle d'évolution indépendant (à paramètres constants) à un modèle d'évolution interactionnel.

\section{Évolutivité des unités d'information}

\section{Concept d'unités d'information}

Bien souvent, une donnée n'est pas productrice de sens par elle-même, mais dépend d'autres données qui lui sont liées sémantiquement.

Par exemple, une image est codée en $N$ lignes * $M$ colonnes, dont chaque cellule $P(n, m)$ est constitué de composantes respectivement Rouge (n, m), Vert (n, m), et Bleu (n, m) du pixel correspondant, codées chacune sur 8 bits (chiffres binaires) dans un système à 16,9 millions de teintes. Un bit isolé n'a pas de sens propre, une composante colorée non plus, un pixel même n'en contient pas davantage Seule une certaine partie est signifiante pour l'utilisateur.

50 Ainsi apparaissent de nécessaires regroupements de données correspondant à des enclipsages signifiants. Ce principe correspond au concept d'unité d'information, dont on peut trouver une définition dans « Hyperdocuments, hypertextes, hypermedia » de JeanPierre Balpe «La découpe de l'information n'est pas une opération neutre. Cette découpe du media, à moins de provoquer une destruction pure et simple de l'information contenue, doit respecter certaines contraintes. Elle n'est possible qu'à partir d'une conception particulière de l'information. Du simple fait de son effectuation, elle définit des unités d'informations discrètes. Une fois définies, celles-ci restent figées pour toute utilisation ultérieure de l'Hyperdocuments. "

\section{Classe d'unités d'information invariantes : Niveau zéro}

51 Ce mode de structuration des données défini par Jean-Pierre Balpe relève de la classe des unités d'information invariantes. 

l'exemple précédent, un détail de l'image peut être à lui seul fortement signifiant. L'usage de données fixes impose alors de réenregistrer une seconde fois le même fragment de données, déjà présent en mémoire, simplement parce qu'il s'agit d'une unité d'information nouvelle. Pour lever cette contrainte, il convient d'introduire d'autres classes d'unités d'informations qualifiées d'unités d'information évolutives.

\section{Classe d'unités d'information évolutives} multiples regroupements ou enclipsages différents sont superposables pour les mêmes données. Ainsi, c'est le nombre et le contenu des unités d'informations qui sont variables dans la classe des données dites évolutives, tandis que les données elles-mêmes demeurent inertes.

L'utilisateur n'est plus cantonné à un rôle de lecteur d'un discours constamment interrompu, découpé en tronçons discontinus d'une manière qui, du reste, ne se justifie pas toujours : il a la possibilité de réutiliser et de réorganiser les unités d'information contenues dans le document.

\section{Unités d'information évolutives insécables liées au contexte : Niveau un}

Dans ce cadre, il y a différents niveaux de complexité. Les deux premiers niveaux concernent les «imités d'information liées au contexte", c'est-à-dire les unités d'information autonomes que l'on peut rassembler indépendamment de tout contexte (comme par exemple une liste classée d'unités d'information), ou d'unités d'information que l'on ne peut utiliser que dans leur contexte initial.

Le premier niveau concerne les unités d'information insécables: on peut les assembler entre elles, mais on ne peut les décomposer afin d'en former de nouvelles. Le cas du «panier d'information» de certains documents entre dans ce cadre. L'utilisateur peut rassembler dans un " panier virtuel » une sélection de page-écran (généralement au sein de documents encyclopédiques), en tout ou rien : il prend tout ou il ne prend pas, mais il ne peut pas découper en fragments plus petits.

\section{Unités d'information évolutives sécables liées au contexte : Niveau deux}

Au second niveau de classification, les unités d'information liées au contexte peuvent être décomposées : par exemple, l'utilisateur peut désolidariser un des objets composant une unité d'information et le réutiliser. Supposons, à titre d'exemple, une application d'initiation à la musique. Pour permettre de discerner parfaitement chacun des instruments de musique, une solution est de donner la possibilité à l'utilisateur d'écouter sur un ou plusieurs morceaux le nombre d'instruments de musique qu'il désire : tous les instruments, tous sauf un, un seul...

\section{Unités d'information évolutives insécables transportables : Niveau trois}

Les deux niveaux supérieurs sont l'extension des deux précédents aux unités d'information évolutives transportables, c'est-à-dire aux unités d'information que l'on peut réutiliser dans des contextes différents du contexte initial. Le niveau trois concerne 
les unités d'information insécables, comme par exemple une application où l'on peut ramasser des petits pois au jardin et les regrouper à la cuisine, dans leur forme initiale.

Unités d'information évolutives sécables transportables : Niveau quatre

Le niveau quatre d'évolutivité est l'extension du niveau trois aux unités d'information sécables. Ceci est le cas dans l'application précédente s'il est possible d'écosser les petits pois dans la cuisine.

\section{Synthèse récapitulative, exemples et perspectives}

\section{Synthèse récapitulative}

Le tableau ci-dessous récapitule les résultats obtenus en adoptant un mode de classification des procédés d'interactivité suivant trois indices: les types de données exploitées par le document, les classes d'unités d'information et les modes scénationnels identifiés dans la production contemporaine.

61 Ce mode de classement ouvre de vastes perspectives. En effet, si chacun peut assimiler les niveaux (zéro, zéro, zéro) à (cinq, zéro, zéro) à des documents de sa connaissance, rien n'interdit la superposition de ces divers niveaux au sein du même document (par exemple en fonction de l'utilisateur). En effet, tous fonctionnent à partir de données fixes, enclipsées dans des unités d'informations invariables. Ce n'est que la structure de liaison qui diffère. On peut sans difficulté de principe superposer plusieurs types de structures aux mêmes données suivant le mode scénationnel retenu.

Classification des niveaux d'interactivité dans un espace trois dimensions.

63 Pour concrétiser les autres niveaux, il est plus facile de les illustrer à partir de titres interactifs significatifs du marché.

\section{Exemples}

Le roi Lion, L'alphabet sauvage et comment ça marche?

Les applications Le roi Lion, L'alphabet sauvage et "Comment ça marche? ont simultanément un niveau zéro de générativité et d'évolutivité. Ces trois applications se situent donc beaucoup plus dans le cadre d'applications à travers lesquelles on "navigue", sans espérer aucune modification tant des unités d'information que des données. "Le roi Lion», document directement inspiré du dessin animé des studios Disney, est ce que l'on pourrait qualifier de «livre interactif», mais avec une forme d'interactivité basique limitée à une navigation indexée matricielle. "L'alphabet sauvage » et "Comment ça marche? ? sont un peu plus évolués dans la mesure où la structure scénationnelle suit un réseau de liens: on accède directement et depuis de multiples endroits à une unité d'information en fonction de critères de sélection. Par exemple pour "Comment ça marche?" (qui est une implémentation interactive d'un livre encyclopédique concernant le fonctionnement de différents appareils et les grands principes de la physique sous-jacents) l'intérêt de ce document, par rapport à sa version "papier» est la possibilité d'entendre des sons et de voir quelques animations (ces dernières peuvent faciliter la compréhension, par exemple le mécanisme de percussion 
d'un marteau sur une corde de piano). La structure de ce document est assez pauvre dans la mesure où les données sont fixes et les unités d'informations invariantes. Si la structure générale du schéma de scénation est d'un niveau quatre (réseau de lien) les parties « animations» ou "diffusions sonores» ne sont que d'un niveau un (linéaire interruptible).

\section{Riven}

Le niveau scénationnel de «Riven » est d'un niveau quatre, en effet, ce jeu est structuré autour de réseaux de liens prédéfinis. Par ailleurs, la générativité des données est d'un niveau zéro (toutes les données sont préexistantes) et l'évolutivité des unités d'information sont d'un niveau un. En effet, certains objets sont réutilisables en tout ou rien dans leur contexte ou sans contexte dans un inventaire.

\section{Versailles, ou complot à la cour du roi soleil}

«Versailles, ou complot à la cour du roi soleil » bien que contenant des données fixes, peut être considéré comme ayant un niveau scénationnel de visite virtuelle par partie au plan visuel, le joueur est placé dans un monde virtuel. Aucune donnée n'est créée, mais certaines unités d'information sont évolutives (en fonction du déroulement du jeu) des objets dans leur globalité, apparaissent dans certaines pièces du jeu, sont réutilisables. Cependant tous ces objets demeurent dans le même contexte ou sont complètement décontextualisés dans un inventaire. L'évolutivité des unités d'information se situe donc au niveau un.

\section{Journeyman Project three : Legacy of time}

"Journeyman Project three: Legacy of time" est comparable à "Versailles", à la différence près que je joueur est complètement plongé dans un espace en trois dimension calculé en temps réel. Cette dernière caractéristique permet à ce jeu d'avoir une générativité de niveau quatre (les données d'affichage sont calculées et sont fonction des actions d'orientation du joueur).

\section{Adibou}

«Adibou » est une application éducative destinée aux jeunes enfants (quatre - six ans). Il est assez original dans la mesure où cette application construit un monde virtuel avec l'habitation d'Adibou et son jardin. Ce qui est particulièrement intéressant ici, c'est le fait que le monde virtuel vit indépendamment des actions extérieures de l'utilisateur.

Pour rester plus terre à terre et classer ce document en fonction des prérogatives fixées, la scénation du document est d'un niveau quatre - la structure de navigation étant une structure indexée en réseau de lien. La générativité des données est aussi d'un niveau quatre puisque tout au long du jeu, les variables d'état de l'évolution du système sont modifiées en fonction des actions du joueur. Ainsi, dans le jardin, si l'enfant arrose régulièrement sa plantation de carottes, celles-ci vont pousser, même si l'enfant ne les regarde pas pousser. Inversement, si le joueur ne s'occupe pas du jardin, les plantations vont dépérir. L'évolutivité est, elle, d'un niveau trois. En effet, différents objets du jeu sont réutilisables dans un nouveau contexte, mais ils le sont en tout ou rien (ces objets, unités d'information ne sont donc pas sécables). Par exemple, l'enfant peut réutiliser les 
ingrédients d'une récolte pour alimenter la réserve à provision (passage du contexte jardin au contexte cuisine).

\section{Blade Runner}

70 Le jeu «Blade Runner », compte tenu de la classification abordée est le plus complexe avec un niveau de visite virtuelle au plan de la scénation, une génération de données « courante utilisateur » et une évolutivité transportable sécable. Le joueur est représenté par un avatar, personnage de synthèse, se déplaçant en temps réel dans un univers en trois dimension. Ainsi, on a bien une navigation en visite virtuelle. Ceci est soutenu par le caractère génératif des données d'une application trois dimensions temps réel, ce qui élève la générativité des données au niveau quatre, comme pour « journeyman ». Enfin, le joueur peut réutiliser des photos ou des vidéos qu'il a préalablement «scanné » sur des zones qu'il a défini sur son parcourt dans un nouveau contexte. Il peut aussi réécouter des rapports, des interrogatoires à la demande. C'est pourquoi le niveau d'évolutivité de ce jeu est au maximum de l'échelle définie avec un niveau quatre.

Bilan

71 La figure ci-après récapitule le classement des différents exemples cités sur un schéma trois dimensions unique. Il donne une première hiérarchie qualitative de différents documents.

Visualisation des niveaux d'interactivité des exemples dans l'espace trois dimensions de classification.

\section{Utilisation de la classification, expérimentation de sa pertinence}

L'usage de la classification est testé avec des étudiants d'IUT en informatique depuis deux ans. La méthode de classification leur est d'abord présentée de manière formelle pendant une heure, lors d'un cours. Ensuite, le vocabulaire typique de la classification est utilisé durant deux heures en groupe de T.D., à travers quatre titres interactifs («L'alphabet Sauvage », «Le Roi Lion », « Versailles » et "Adibou »). La dernière partie de l'exercice consiste, pour les étudiants, à écrire un rapport de synthèse sur les présentations des documents cités.

Nous ne disposons pas aujourd'hui d'une étude quantitative sur les rapports des étudiants, cependant plusieurs remarques peuvent être faites. Le classement en mode de scénation ne pose pas de problème, sachant que la terminologie liée à la structure du document (linéaire, indexé...) est préférée à celle liée aux activités (audiovision, consultation...). En ce qui concerne le classement de générativité des données et d'évolutivité des unités d'information, les réactions des étudiants face à ces concepts ont amené la décomposition de ces critères qui jusque-là étaient traités de façon binaires (données fixes/génératives, unités d'information invariantes/évolutives). Par conséquent, la pertinence et la transférabilité du modèle sont confortées par l'expérimentation. 


\section{Perspectives}

En l'état, la méthode proposée permet tout d'abord une identification claire des procédés d'interactivité que comporte un document achevé ou à naître, et permet ainsi une certaine évaluation qualité des documents interactifs. Les exemples cités ont permis d'établir un premier classement de documents en fonction du maximum d'interactivité perçu. Cependant cette classification demeure ponctuelle, les critères maxima ne s'appliquent pas à l'intégralité des documents.

En effet, toutes les musiques de ces applications sont en audio vision, tout comme les sons parlés dans « Le roi Lion ", « l'alphabet sauvage » et "Comment ça marche? ». De même, si on retire l'aspect immersion trois dimensions temps réel de Legacy of time, Versailles et ce dernier seraient classés au même niveau (si la technologie l'avait permis, Versailles serait probablement en immersion trois dimensions temps réel). Mais, ces deux jeux n'ont pas le même intérêt Riven est très mal classé alors que son intérêt ludique est très important.

Les critères adoptés ne sont donc, à ce stade, pas suffisants pour juger complètement et objectivement la qualité des applications multimédia.

Peut-on, pour un document, considérer comme référence absolue, le classement maximal obtenu par un élément précis du document ? Faut-il, au contraire considérer uniquement une moyenne de classification? En fait, ni l'une ni l'autre des deux hypothèses, employée seule, ne semble convenir. Peut-être faut-il passer par une représentation des différents classements sur un schéma en trois dimensions (tel qu'il a été présenté à la figure un) avec des densités de classification. La réflexion sur ce domaine est encore à approfondir.

Par ailleurs, pour comparer Riven, Versailles et Legacy of time, si on évaluait les macro structures rhétoriques, c'est-à-dire le mode d'exposition des éléments de l'application à l'utilisateur, on obtiendrait de nouveaux critères de classement qui réévalueraient l'intérêt de certaines applications.

80 En résumé, cette méthode apporte de nouveaux outils pour l'évaluation qualité des documents interactifs et ouvre la voie à des perspectives de développement intéressantes. En tout état de cause, outre l'identification des procédés d'interactivité, elle les relie ensuite à des schémas de structuration précis facilitant la programmation et le développement. Elle permet enfin de canaliser le champ exploratoire des recherches futures vers des types de documents quasiment inconnus à ce jour.

\section{BIBLIOGRAPHIE}

Normes pour le management de la qualité et l'assurance qualité, partie I, Lignes directrices pour leur sélection et leur utilisation, transformation de la norme française NF X 50-121-1 en norme européenne ISO 9000-1,1994.

BALPE J.-P, Hyper documents, hypertextes, hypermedia, Paris, Eyrolles, 1990. 
BALPE J.-P, Un roman inachevé Littérature n96, université de Paris VIII Vincennes-Saint-Denis, Larousse, Paris, 1995.

BALPE J.-P, À propos d'un roman inachevé, propos recueillis par Olivier Kœchlin Dossier 1995 de l'audiovisuel $n{ }^{\circ} 64$, INA, Bry sur Marne.

COLAITIS F, FROMONT j., KRETZ F., Évolution of multimedia interactive applications towards hypermedia, Rennes, Document Woodman 89, BIGRE-IRISA, 1989.

COLIN M., Cinéma, télévision, cognition, Nancy, Presses Universitaires, Collection « Processus Discursifs-Langage et Cognition », 1992.

CONKLIN J., Hypertext : an introduction and survey, Computer I.E.E.E., septembre 1987.

DURAND A., La modélisation moléculaire : vers un modèle de conception multimédia, Thèse de doctorat, Université de Valenciennes et du Hainaut Cambrésis.

EISENSTEIN S.-M., Dramaturgie de la forme filmique, Paris, Dunod, 1929.

Joannis H., De l'étude de la motivation à la création publicitaire, Paris, Dunod, 1965.

LELEU-MÉRVIEL S., La scénistique : méthodologie pour la conception de documents en media multiples suivant une approche qualité, Université de Paris 8 , Thèse pour l'habilitation à Diriger des Recherches, 1996.

LELEU-MÉRVIEL S., La conception en communication : méthodologie qualité, Éditions Hermès, 1997.

LELEU- MÉRVIEL S., Le scénario multi media, Paris, Hermès, à paraître en 1999.

NIGEAY L., COUTAZ J., Espaces conceptuels pour l'interaction multimedia et multimodale, Paris, Hermès, Revue internationale Technique et science informatiques, Rairo Afcet, vol. 15, n 9, 1996, 1195-1225,1996.

POGNANT P., Scholl C, Les CD-Rom culturels, Paris, Hermès, 1996. Souriau E., L'univers filmique, Flammarion, 1953.

\section{RÉSUMÉS}

La qualité désigne la capacité d'une entité à répondre aux besoins de son client potentiel. L'élément-clef d'une communication/produit de qualité doit permettre au client d'évaluer, avant achat, la capacité du produit à satisfaire ses attentes. S'agissant des produits multimédia, peu d'informations fiables sont fournies sur les caractéristiques de l'objet proposé. Celles-ci définissent au mieux un nombre de fragments inertes sans estimation des capacités d'interaction. Cet article propose un principe de classification des procédés d'interactivité. Il se fonde sur la nature des données, une forme de structure supportant leur architecture et les unités d'information du document. Il permet de classer une application interactive. Des exemples d'applications illustrent les résultats de la méthode pour un ensemble de titres significatifs du marché.

Quality refers to the ability of an entity to meet potential customer needs. The key element of a quality-based communication/product is its ability to allow a customer to evaluate its capacity to satisfy his needs prior to its purchase. Regarding multimedia products, very little information is given concerning the specifications of the object in question. These details define, at best, only a certain number of passive fragments without evaluating its interactive capacities. This article proposes a system of classification regarding interactive processes. It is based on the nature of the data, the pattern of the structure underlying its architecture and the units of information of 
a given document. This facilitates the classification of multimedia documents. Some examples illustrate the method by using some major products currently available.

\section{INDEX}

Mots-clés : interactivité, niveaux d'interactivité, classification, document, multimédia

\section{AUTEURS}

\section{JEAN-MARC LAUBIN}

Jean-Marc Laubin est professeur associé à l'Université de Valenciennes (UVHC). Indépendant en développement d'outils informatiques pour la production audiovisuelle et multimédia, il préside la commission multimédia de la CST (Commission Supérieure Technique du Centre National de la Cinématographie).

\section{ALAIN DURAND}

Alain Durand est maître de conférences à l'UVHC et travaille sur le scénario multimédia.

\section{SYLVIE LELEU-MERVIEL}

Sylvie Leleu-Merviel est professeur et dirige le Laboratoire des Sciences de la Communication de l'UVHC. Ses recherches concernent l'ingénierie du document 